\title{
Cognitive control training as an augmentation strategy to CBT in the treatment of fear of failure in undergraduates
}

\author{
Nathan Van den Bergh ${ }^{1}$, Sarah Vermeersch ${ }^{2}$, Kristof Hoorelbeke ${ }^{3}$ Jasmien Vervaeke $^{4,7}$, \\ Rudi De Raedt ${ }^{5} \&$ Ernst H.W. Koster ${ }^{6}$
}

\footnotetext{
1, 3-6 Department of Experimental Clinical and Health Psychology, Ghent University, Ghent, Belgium

${ }^{2}$ Student Counseling Office, Department of Educational Policy, Ghent University, Ghent, Belgium

7 imec-mict-UGent, Ghent University, Ghent, Belgium
}

Corresponding author:

Department of Experimental Clinical and Health Psychology

Ghent University

Henri Dunantlaan 2

B-9000 Gent, Belgium

+32 9-264-94-14

e-mail: Nathan.VandenBergh@UGent.be

\footnotetext{
${ }^{1}$ https://orcid.org/0000-0002-5592-6682

Twitter: @Nathan_VdBergh

${ }^{3}$ https://orcid.org/0000-0002-8269-0441

${ }^{4}$ https://orcid.org/0000-0002-3336-1887

Twitter: @JasmienVervaeke

${ }^{5}$ https://orcid.org/0000-0001-6781-4808

${ }^{6}$ https://orcid.org/0000-0003-0792-476X

Twitter: @ernst_koster
} 


\begin{abstract}
Background. Previous research in the context of depression indicates that Cognitive Control Training (CCT) has the potential to reduce maladaptive emotion regulation, such as rumination. However, as a stand-alone intervention, CCT does not seem to increase adaptive emotion regulation. We examined whether CCT combined with a traditional fear of failure intervention program would improve emotion regulation and psychopathology symptoms.

Methods. 102 students participating in a group-based cognitive behavior therapy (CBT) program targeting fear of failure were randomized to CCT or active placebo conditions, performing ten 15-minute sessions of the adaptive Paced Auditory Serial Addition Task or an adaptive speed-ofresponse task, respectively. Primary outcome measures were Repetitive Negative Thinking (RNT) and symptoms related to depression, anxiety and stress. Secondary outcomes included adaptive cognitive emotion regulation strategies.
\end{abstract}

Results. Task-specific cognitive transfer was observed in the CCT condition. In both conditions, RNT and symptoms were reduced. In contrast to our hypotheses, the CCT condition did not significantly differ from the active control condition in terms of treatment effects.

Conclusions. The current study is among the first to investigate the added value of combining CCT with CBT in an anxious sample. CCT did not augment effects of a CBT-based fear of failure treatment. 
Cognitive control refers to the ability to flexibly adapt thoughts and behavior as a function of one's goals (Cohen, 2017; Miller \& Cohen, 2001). Impairments at the level of cognitive control have been proposed as a transdiagnostic risk factor for internalizing disorders such as anxiety and depression (Goschke, 2014; Hankin et al., 2016; Snyder et al., 2015). For anxiety in particular, recent meta-analytic evidence based on 58 studies $(N=8292)$ revealed anxiety-related deficits in specific cognitive control processes (Shi et al., 2019). Crucially, these deficits at the level of cognitive control have implications for emotion regulation. In particular Repetitive Negative Thinking (RNT), a transdiagnostic risk factor overarching processes such as rumination and worry (Ehring \& Watkins, 2008), forms a key concept in understanding the relation between cognitive control and psychopathology. An abundance of evidence (Drost et al., 2014; Klemanski et al., 2017; McEvoy et al., 2013; Spinhoven et al., 2015) supports the role of RNT in the co-occurrence of depression and anxiety, with RNT being linked to cognitive control deficits in the context of depression (Joormann \& D'Avanzato, 2010; Koster et al., 2011), anxiety (Hirsch \& Mathews, 2012) or both (Zetsche et al., 2018).

More specifically, researchers theorize that cognitive control deficits hamper the ability to interrupt maladaptive emotion regulation processes, such as RNT, at the expense of alternative emotion regulation strategies, such as reappraisal or problem solving. Very recently, the impact of executive functioning on rumination, as formulated by the impaired disengagement hypothesis (Koster et al., 2011), has been included in the H-EX-A-GO-N model, an integrated theoretical framework for rumination (Watkins \& Roberts, 2020). This theory argues that reduced cognitive control is an important factor in increasing the susceptibility for RNT. A number of prospective studies support this idea (e.g., De Lissnyder et al., 2012). Although there is some evidence for relationships between RNT and specific aspects of cognitive control (e.g., Zetsche et al., 2018), other authors have suggested that fractionating cognitive control is not the most fruitful avenue for research on psychopathology and cognitive control (Grahek et al., 2018).

Importantly, experimental studies (Philippot \& Brutoux, 2008; Sari et al., 2017; Watkins \& Brown, 2002) have also found evidence for the inverse relation: a negative impact of RNT on cognitive control. These findings are in line with Attentional Control Theory (Eysenck et al., 2007), 
stating that anxiety causes increased allocation of attention to threat-related stimuli, including negative thoughts, leaving less attentional resources for goal-directed functioning. In addition, impaired cognitive control increases the risk for stressful life events, such as interpersonal conflict and academic failure, triggering RNT (Snyder \& Hankin, 2016). Taken together, these findings suggest the risk of a vicious cycle: cognitive control deficits directly and indirectly set the stage for RNT, which in turn increases cognitive load, making it harder to successfully disengage (cf. Koster et al., 2011) from RNT.

Research on Cognitive Control Training (CCT), consisting of repeated performance of a computerized task targeting executive functions, suggests a causal role of cognitive control in RNT. A frequently used training procedure is the adaptive Paced Auditory Serial Addition Task (PASAT) (Gronwall, 1977; Siegle et al., 2007). In the adaptive PASAT, participants are required to keep track of a continuous auditory stream of stimuli (i.e., digits), in order to manipulate (i.e., add up) these stimuli in working memory, whilst resisting distractions. These distractions include no longer relevant digits/sums, negative thoughts about one's own performance and stress related to an increasing pace and errors. Studies comparing ten 15-minute sessions of the adaptive PASAT with a control task have shown that the adaptive PASAT, but not the control task, can buffer against RNT in samples of undergraduates reporting high levels of RNT, when confronted with stressors in the lab (Hoorelbeke et al., 2015) or daily life (Hoorelbeke et al., 2016; Hoorelbeke et al., 2015).

Other modifications of classic experimental tasks have also been used as CCT, including Flanker (e.g., Cohen et al., 2015; Cohen et al., 2016) and Dual n-back (e.g., Onraedt \& Koster, 2014; Schweizer et al., 2013; Wanmaker et al., 2015) tasks. In some studies, the data not only supports the hypothesis that CCT can reduce RNT (Cohen et al., 2015), but also that it can have effects at the neural level (Owens et al., 2013; Schweizer et al., 2013). Other studies, however, have not been able to find beneficial effects with these types of CCT (Onraedt \& Koster, 2014; Wanmaker et al., 2015). For an extensive overview of CCT in the context of depression, the reader is referred to a systematic review of Koster and colleagues (2017), where it was noted that the PASAT has the most consistent empirical support as CCT. 
Although there is evidence that the PASAT can reduce RNT, several studies have observed that, as a standalone intervention, it does not increase the use of adaptive emotion regulation strategies (Hoorelbeke \& Koster, 2017; Hoorelbeke et al., 2016). Interestingly, it has been suggested in this context that, through an increased ability to down-regulate negative thoughts and feelings, CCT may increase the effectiveness of existing therapeutic programs that promote the use of adaptive emotion regulation strategies (Van den Bergh et al., 2018).

The amount of studies investigating CCT as treatment augmentation is limited. Of particular interest is a study comparing the effectiveness of CCT and mindfulness as standalone interventions with a control group and a group receiving a combination of CCT and mindfulness, in a sample of participants reporting high levels of RNT (Course-Choi et al., 2017). The results indicated that a continued decrease in RNT one week after completion of the intervention was only present in the combined condition, suggesting that CCT (operationalized using an adaptive dual n-back task) may partly tap into similar attentional processes as mindfulness (Course-Choi et al., 2017). Additionally, it has been observed that combining CCT with treatment as usual in the context of depression resulted in additional benefits in terms of rumination and sustained treatment gains (Siegle et al., 2014). However, other studies have yielded less promising findings. For instance, combining CCT with behavioral activation did not improve treatment effects (Moshier \& Otto, 2017). As such, continued research on the potential of CCT as a means to augment effects of existing treatments is necessary. Furthermore, CCT may be of particular interest when combined with other interventions that target emotion regulation processes.

The current study set out to investigate the potential of CCT as treatment augmentation in a sample that experiences significant levels of RNT. More specifically, a university-based CBT program targeting fear of failure was supplemented with either CCT, which was operationalized using the adaptive PASAT, or a control training. The abovementioned fear of failure program is offered to undergraduates, a population that has an increased risk for developing mood, anxiety and substance use disorders (Auerbach et al., 2018). More specifically, a significant amount of undergraduates report test anxiety (Gerwing et al., 2015), in which RNT plays a central role as well: (1) it may be triggered 
when accessing negative self-beliefs (relating to personal incompetence, for instance), (2) it can hamper performance in test situations, and (3) it can further strengthen the negative self-beliefs (Zeidner \& Mathews, 2005).

Given the impact of anxiety on executive functioning (Eysenck et al., 2007), the role of executive functioning in worry (Hirsch \& Mathews, 2012) and -crucially- the notion that executive functioning is required to successfully disengage from negative thoughts (Koster et al., 2011), we hypothesized a more pronounced decrease in RNT in the experimental condition (i.e., the condition in which participants carry out the adaptive PASAT) as compared to the control condition. Moreover, we also expected that symptoms related to depression, anxiety and stress would also improve to a greater extent in the experimental condition, an effect that may be mediated by the reduction in RNT. The secondary outcome measures included various other adaptive (e.g., refocusing on planning) and maladaptive (e.g., catastrophizing) cognitive emotion regulation strategies, as well as effortful control (Derryberry \& Rothbart, 1988), a concept that is strongly related to cognitive control. These hypotheses were tested in a single blind randomized controlled trial, and were preregistered on the Open Science Framework (https://osf.io/7xmwn, see study 2).

\section{Method}

\section{Participants}

Ghent University students who enrolled in a group program targeting fear of failure between October 2017 and April 2019 were invited to participate in the study. A total of 102 students (see Figure 1: CONSORT diagram) provided informed consent and were randomized to the CCT or active placebo condition. Eight participants did not start with the baseline phase after receiving the necessary information to log in on the training platform. An additional five participants started but did not complete the baseline phase, resulting in a final sample of 89 participants. Participants were aged 18 to $28(M=20.78, S D=2.28)$, mostly female $(N=71$ or $79.7 \%)$ and diverse in terms of their fields of study. The majority of the participants $(N=56$ or $62.9 \%)$ reported a history of psychotherapy. The 
project was approved by the medical ethical committee of Ghent University. Following completion of the follow-up questionnaires, participants received a monetary reimbursement of $€ 15$.

\section{Design}

The participants in both conditions took part in the fear of failure program and were randomly allocated to either the experimental condition or the control condition by the first author, based on signed informed consent forms coming in. Measurements were obtained at three different time points: before the start of the online training, shortly after treatment completion (i.e., all online training sessions as well as all group sessions) and at two months follow-up. The participating students did not know to which condition they were randomized, and neither did the therapists who guided the group program. The researchers, on the other hand, were aware of the group allocation. Except for a brief introductory visit by the first author at the end of the first group session and standardized mails during the course of the study, there was no contact between the researchers and the students.

\section{Fear of Failure Program}

The fear of failure program is a group-based program (with a maximum of 12 students per group, provided by student counselors. Based on an individual intake interview with a student counselor, where there is an assessment on the severity of the problems, people can be included in the course with five weekly 120 -minute sessions, if fear of failure is moderate to severe. Content-wise, the sessions are based on CBT-techniques and structured as follows:

1. Psycho-education on fear of failure and coping strategies (e.g., contrasting avoidance with constructive action), in order to help the students identify their own tendencies.

2. Psycho-education on productive versus counter-productive study habits, in order to help the students identify and adapt their own method. 
3. Strategies to deal with negative thoughts, such as confining negative thinking to a limited time and a specific place, spotting thinking errors and phrasing alternatives, disentangling observations from interpretations, accepting the presence of negative thoughts without responding in counterproductive ways,...

4. Relaxation strategies (e.g., breathing exercises).

5. Strategies to deal with negative thoughts: further elaboration and closing.

\section{Online Training Tasks}

Participants who were randomized to the experimental condition performed ten sessions of the adaptive PASAT. In this task, participants are presented with an auditory stream of digits (between 1 and 9) and a visual array of numbers (between 1 and 18). Participants are instructed to continuously respond to the sum of the last two heard digits by clicking (desktop/laptop computer) or tapping (tablet computer) on the corresponding number on the visual array as quickly and accurately as possible. For every new digit that is presented, a new sum has to be calculated and selected on the screen of the participant's device. Crucially, the inter-trial interval (ITI) changes depending on the participant's performance: for every four consecutively correct responses, the time between the auditory stimuli decreases in increments of $100 \mathrm{~ms}$, resulting in an increased pace. In case of four consecutive incorrect responses, the pace decreases in increments of $100 \mathrm{~ms}$, decreasing the speed of the task. The participants' performance on a session helped determine the starting ITI of the subsequent session. In earlier descriptions of the above procedure (Van den Bergh et al., 2018) it has been suggested that the task demands recruit attentional control mechanisms of working memory (Shipstead et al., 2014) and updating (Miyake et al., 2000). Insofar that the participant is prone to negative thoughts (e.g., relating to failure), he or she may also have to continuously inhibit the intrusion of such thoughts (Anderson et al., 2016) or discard them from working memory (Zetsche et al., 2018) while carrying out the task itself.

In the control condition, participants are presented with the same visual array of numbers between 1 and 18 as in the experimental condition, which is in line with previous research 
(Hoorelbeke \& Koster, 2017). Unlike participants in the experimental condition however, participants in the control condition hear numbers between 1 and 18 (rather than 1 and 9), and they simply have to tap or click on the corresponding number, without summation. Again, performance influences the ITI, with the pace increasing, decreasing or remaining the same, depending on the response pattern. As such, this procedure can be described as an adaptive speed-of-response task. As opposed to the adaptive PASAT, participants do not need to continuously keep track of the previous digit, calculate sums and withstand the same level of task-related interference (e.g., the previous sum or digit). Although the adaptive speed-of-response task is cognitively less demanding than the PASAT, it is unlikely to be a sham intervention, as participants still have to put effort into sustaining their attention, in the face of frequent errors. The adaptive speed-of-response task will be referred to as the Active Control Training (ACT).

\section{Measurements}

Credibility/Expectancy Questionnaire. The Dutch version of the Credibility/Expectancy Questionnaire (CEQ) (Devilly \& Borkovec, 2000; Godfrin et al., 2004) was used to check for differences between conditions in credibility and expectancy, with regards to the online training. To avoid confusion, the wording of some of the items was slightly modified to clarify that only the online training, rather than the intervention as a whole (i.e., including the fear of failure program), had to be taken into account when answering the questions. An additional version with modified wording was created for use after the intervention. The CEQ consists of 6 items that measure the degree to which participants think and/or feel that the intervention is logical, helpful and worth recommending to a friend. Depending on the question, participants have to answer using a 9-point Likert scale (1: "not at all logical/confident/useful", to 9: "very logical/confident/useful”) or indicate how much symptom improvement they expect ( $0 \%$ to $100 \%$, with increments of $10 \%)$. Analyses of the CEQ will be carried out using z-scores. 
Depression, anxiety and stress symptoms. The shortened (Henry \& Crawford, 2005) version of the Dutch translation of the Depression Anxiety Stress Scale (DASS) (de Beurs et al., 2001; Lovibond \& Lovibond, 1995) consists of 21 items intended to cover a wide range of the key symptoms related to depression, anxiety and stress ( 7 items each). Some of the included items are 'I couldn't seem to experience any positive feeling at all" (depression), "I felt scared without any good reason" (anxiety) and "I found it difficult to relax" (stress). Participants are asked to take into account the past week when responding to the items, using a 4-point Likert-scale, ranging from 0 ("Did not apply to me at all") to 3 ("Applied to me very much, or most of the time").

Repetitive negative thinking. The Dutch version of the Perseverative Thinking Questionnaire (PTQ) (Ehring et al., 2012; Ehring et al., 2011) was selected to quantify RNT, as it is a measure of shared processes in worry and rumination. The PTQ consists of 15 items, that are equally spread across three core and two additional characteristics of repetitive negative thinking. The core characteristics refer to the repetitive and intrusive nature of negative thoughts, as well as the experienced difficulty to disengage from negative thoughts ( 3 items each). The additional characteristics refer to the impact of the thinking process, such as the extent to which it captures mental capacity (3 items) while not necessarily contributing to a solution (i.e., perceived unproductiveness, 3 items). For each of the items, participants indicate to what degree the statement applies to them, when they are thinking about negative experiences or problems, using a 5-point Likert-scale (ranging from 0: “never”, to 4: "almost always”).

Cognitive emotion regulation strategies. The original Dutch version of the Cognitive Emotion Regulation Questionnaire (CERQ) (Garnefski et al., 2001) was used to measure adaptive as well as maladaptive emotion regulation strategies. The maladaptive strategies are self-blaming, rumination, catastrophizing and blaming others. The adaptive strategies are acceptance, refocusing on the positive, refocusing on planning, positive reappraisal and putting into perspective. Each of these 9 strategies are quantified using 4 items, for a total of 36 items. Participants are asked to indicate on a 5point Likert-scale (ranging from 1: “(almost) never”, to 5: "(almost) always") to what extent they typically experience certain thoughts upon confrontation with negative experiences. In line with earlier 
research (Vanderhasselt et al., 2014), compound scores for adaptive and maladaptive strategies will be calculated separately and used for the analyses.

Effortful control. The Dutch version of the Effortful Control (EC) factor of the Adult Temperament Questionnaire (ATQ) (Derryberry \& Rothbart, 1988; Hartman et al., 2001) was used. Items related to the EC factor measure one's self-reported capacity to regulate attention and behavior as a function of one's goals. As such, the instrument likely taps into a subjective aspect of cognitive control. The EC factor consists of 19 items, each quantifying one of the following three functions: Activation Control (7 items, e.g., "I can keep performing a task even when I would rather not do it"), Attentional Control (5 items, e.g., "When interrupted or distracted, I usually can easily shift my attention back to whatever I was doing before") and Inhibitory Control (7 items, e.g., "Even when I feel energized, I can usually sit still without much trouble if it's necessary"). Participants respond to each item using a 7-point Likert-scale, ranging from 1 ("Extremely untrue") to 7 ("Extremely true"), while also having the option to answer with X ("Not applicable").

Task-specific cognitive transfer. The non-adaptive PASAT was used at baseline and as part of the post-training measurements, to check for the progress that participants had made. The nonadaptive PASAT consists of three blocks of 60 trials each (ITIs of $3000 \mathrm{~ms}, 2000 \mathrm{~ms}$, and $1500 \mathrm{~ms}$, respectively). To minimize the risk that participants did not (fully) understand the task before getting started, they were guided through multiple levels of instructions: a short video-clip in addition to a written explanation (summarized), followed by another visual example and five practice trials. At the end of each block, participants could take a brief pause before starting the next. All 180 trials in total require 6.5 minutes, not taking into account the time needed for the instructions, nor the short breaks participants could take in between blocks. Performance on the non-adaptive PASAT is indexed as the percentage of correct trials across all blocks.

\section{Procedure}

At the end of the first session of the fear of failure program, the first author visited each of the eighteen groups that were organized between October 2017 and September 2019. During this visit, the 
researcher introduced the study and handed out informational letters, including informed consents.

Signed informed consents were collected by the therapist at the beginning of the second session of the fear of failure program, and handed over to the researcher shortly thereafter. Then, the researcher added the participants' e-mail addresses to the online platform, which automatically assigns participants to either the Cognitive Control Training (CCT, Adaptive PASAT) or the Active Control Training (ACT, Adaptive Speed of Response Task). The participants were provided all necessary information to log in on the study's online platform (e.g., a randomly generated password) via e-mail.

As soon as participants logged on to their accounts, they were presented with the digital version of the informed consent and the terms of service of the online platform. After formally accepting these terms of service, participants started with the baseline measures, consisting of all of the abovementioned questionnaires, followed by the non-adaptive PASAT. After completing the baseline measures, participants were required to carry out the training: ten 15-minute sessions (CCT or ACT), over a period of two weeks. Participants could freely plan these sessions, though they were asked to strive for an optimal spread (i.e., a maximum of one session per day with a rest day every couple of sessions). Upon completion of the tenth and final online training session, participants were informed that they were in the waiting phase until the last session of the fear of failure program had taken place. As such, the post-measurements were planned after the entire intervention (i.e., both the online training and the group program) was completed. These post-measurements consisted of the same questionnaires as the baseline measures, again followed by the non-adaptive PASAT. Finally, two months after the post-training assessment, participants were asked to complete the follow-up questionnaires, which included the DASS, PTQ, CERQ and ATQ (EC), but not the CEQ.

\section{Statistical Analyses}

Analyses are based on all participants who completed the baseline measures $(\mathrm{N}=89)$. Following the intention-to-treat principle, the last known scores of participants that were lost to follow-up (17 participants or $19.1 \%$ ) were carried forward. Most dropout (15 out of 17) occurred before or during the 
online training phase ( 7 in the ACT condition versus 8 in the CCT condition). The training effects are tested using repeated measures ANOVAs followed-up with planned t-tests, and further investigated with Bayesian independent-samples t-tests. As opposed to the frequentist approach, the Bayesian approach allows the estimation of the accumulation of evidence for the null and alternative hypotheses. As such, post-hoc Bayesian analyses provide the opportunity to determine how confident one can be about observed differences in the outcome measures, or lack there-of, between conditions. More specifically, one-sided Bayesian independent-samples t-tests of delta scores will be used to compare the change in scores over time between conditions. All analyses were performed using the JASP software (version 0.8.4.0), with the exception of $d_{\mathrm{av}}$, (Lakens, 2013), which was calculated using version 5 of Lakens' spreadsheet-based calculator (https://osf.io/vbdah/).

\section{Results}

\section{Group Characteristics}

Participants in the CCT and ACT conditions did not show baseline differences concerning demographic variables (age and gender), educational variables (e.g., current study level), or variables related to past use of mental health care (see Table 1). With the exception of one $\mathrm{PhD}$ student in the CCT condition, all participants were students following courses at bachelor or master level. Of the 65 participants that were pursuing a bachelor degree, eight were enrolled at a university college, whereas the remainder enrolled at university $(N=57)$. In addition, there were no differences between conditions in the occurrence of missed sessions in the group program, nor were there differences in the timing of the online training and measurements.

In Table 2, means and standard deviations for all outcome measures are reported, per condition and per time point. Specifically for RNT at baseline, for instance, the mean PTQ scores (out of 60) were high in both the CCT (40.1, SD = 9.3) and ACT (42.6, SD = 9.9) conditions, and consistent with clinical rather than healthy comparison groups (Ehring et al., 2011; Ehring et al., 2012). Similarly, mean symptom levels (DASS-21) at baseline were noticeably higher than in healthy samples (Henry \& Crawford, 2005; Osman et al., 2012) and in line with the mean scores (DASS-42) of various clinical 
groups (Antony et al., 1998). Taken together, these data suggests that there were considerable levels of psychopathology in our sample.

\section{Training Progress}

Progress on the training task. As in previous research (Hoorelbeke \& Koster, 2017), the participants' performance during training was evaluated using the median Inter-Stimulus Interval (ISI) of each session. Participants repeatedly carrying out their training tasks, should improve on the task (i.e., show a decrease in median ISI). Given that the task demands of the CCT and the ACT are very different, separate repeated measures ANOVAs were carried out for each task, to check whether participants are indeed able to reach a higher pace as they complete more sessions. As expected, both groups showed a significant and large improvement in performance (CCT: Greenhouse-Geisser corrected $F(3.64,138.36)=375.88, p<.001, \eta_{p}{ }^{2}=0.91$; ACT: Greenhouse-Geisser corrected $F(2.13$, $\left.72.52)=169.12, p<.001, \eta_{p}{ }^{2}=0.83\right)$.

Perception of the training as an intervention. Results of the repeated measures ANOVAs of the Credibility and Expectancy scores (CEQ) indicated that there are no effects of time, group or the interaction between time and group (see Table 3). Put differently, the perception of the online training did not significantly differ between groups, nor did it change as participants repeatedly carry out the task. As such, there are no indications that participant blinding was unsuccessful. The means of the individual items suggest moderate levels of credibility and (positive) expectancy at best, whereas the expected percentage of symptom reduction (both in terms of thinking and feeling) were relatively high (i.e., around 40\%), but with a high amount of interpersonal variability (see Supplemental Material 1).

\section{Repeated Measures ANOVAs}

Task-specific cognitive transfer. Mean accuracy on the non-adaptive version of the PASAT was around $30 \%$ in both conditions at baseline, while there was a clear mean difference of almost $25 \%$ 
between conditions following the intervention (see Table 2). Indeed, the repeated measures ANOVA suggests a differential effect of the online training procedure, as there is a significant interaction effect between time and group (see Table 3). Post hoc paired samples $t$-tests reveal that participants in both conditions significantly improved on the non-adaptive PASAT (CCT: $t(46)=13.14, p<.001, d_{a v}=2.28$; ACT: $\left.t(41)=8.38, p<.001, d_{a v}=1.01\right)^{1}$. Importantly, independent-samples $t$-tests confirm that participants in the CCT condition significantly outperformed participants in the ACT condition after completion of the intervention $\left(t(87)=6.34, p<.001, d_{s}=1.35\right)$, while no such difference was present at baseline $\left(t(87)=0.71, p=.477, d_{s}=0.15\right)$.

Outcome measures. The repeated measures ANOVAs of the DASS and PTQ (i.e., the primary outcome measures) consistently show only a main effect of time (see Table 3 , all $p$-values <.001). Planned paired samples $t$-tests revealed that, across both conditions, DASS and PTQ scores consistently decrease from baseline to post (PTQ total: $t(88)=5.78, p<.001, d_{a v}=0.50$; DASS depression: $t(88)=5.44, p<.001, d_{a v}=0.43$; DASS anxiety: $t(88)=4.51, p<.001, d_{a v}=0.41$; DASS stress: $\left.t(88)=4.87, p<.001, d_{a v}=0.43\right)$ and from baseline to follow-up (PTQ total: $t(88)=8.14, p<.001$, $d_{a v}=0.70$; DASS depression: $t(88)=5.03, p<.001, d_{a v}=0.48$; DASS anxiety: $t(88)=5.63, p<.001$, $d_{a v}=0.54$; DASS stress: $\left.t(88)=4.65, p<.001, d_{a v}=0.44\right)$. For the PTQ scores, there was a continued decrease from post to follow-up $\left(t(88)=2.91, p<.01, d_{a v}=0.20\right)$, while the DASS scores remained stable in the period following the intervention (DASS depression: $t(88)=0.29, p=.772, d_{a v}=0.03$; DASS anxiety: $t(88)=1.35, p=.180, d_{a v}=0.13$; DASS stress: $\left.t(88)=0.02, p=.980, d_{a v}=0.00\right)$. There is no evidence for the hypothesized interaction effect between time and group, suggesting that the online training procedures (CCT versus ACT) do not have a differential impact on RNT or symptoms related to depression, stress and anxiety (all $F \mathrm{~s}<1)$.

Similarly, the adaptive and maladaptive components of the CERQ, a secondary outcome measure, show the same pattern of results: only a main effect of time $(p<.001$, see Table 3$)$, which again

\footnotetext{
${ }^{1}$ Effects of (potential) violations of test assumptions due to the distribution of variables were evaluated using non-parametric tests. However, the main analysis proved to be robust, adding to the validity of the reported findings.
} 
suggests that the CCT and ACT conditions do not provide differential effects ${ }^{2}$. Planned paired samples $t$-tests revealed that, across both conditions, the maladaptive compound score decreased from baseline to post $\left(t(88)=5.35, p<.001, d_{a v}=0.38\right)$ and from baseline to follow-up $\left(t(88)=6.28, p<.001, d_{a v}=0.48\right)$, while remaining stable from post to follow-up $\left(t(88)=1.38, p=.172, d_{a v}=0.09\right)$. Conversely, the adaptive compound score increased from baseline to post $\left(t(88)=3.78, p<.001, d_{a v}=0.28\right)$ and from baseline to follow-up $\left(t(88)=3.46, p<.001, d_{a v}=0.31\right)$, while remaining stable from post to follow-up $\left(t(88)=0.46, p=.649, d_{a v}=0.03\right)$.

Finally, as part of the secondary outcome measures, the results of the EC factor of the ATQ (see Table 3) revealed a significant main effect of group $(p<.01)$, on top of the main effect of time $(p<.001)$. Post hoc independent-samples $t$-tests indicated that participants in the CCT condition tended to rate their EC significantly higher than participants in the ACT condition, on all time points (Baseline: $t(87)=2.13, p<.05, d_{s}=0.45$; Post-training: $t(87)=2.92, p<.01, d_{s}=0.62$; Follow-up: $t(87)=3.02, p<.01$, $\left.d_{s}=0.64\right)$. Planned paired samples $t$-tests revealed that, across both conditions, EC increased for all three comparisons (baseline to post: $t(88)=4.03, p<.001, d_{a v}=0.24$; baseline to follow-up: $t(88)=6.79$, $p<.001, d_{a v}=0.42$; post to follow-up: $\left.t(88)=3.21, p<.01, d_{a v}=0.17\right)$.

\section{Post-hoc Bayesian Analyses}

Not rejecting the null hypothesis using the frequentist approach indicates a lack of evidence for the alternative hypothesis. However, this does not allow estimation of evidence supporting the null hypothesis (Kruschke, 2011; Mulder \& Wagenmakers, 2016). As such, to further investigate the null findings for the interaction effects of the repeated measures ANOVAs, post-hoc Bayesian independent-samples $t$-tests were carried out. These allow to investigate whether our data is more likely under the null hypothesis, as compared to the likelihood of the data given that the alternative hypothesis is true. If the data is indeed more likely under the null hypothesis, then the evidence against

${ }^{2}$ As preregistered, we hypothesized that the differential effects of training on depression, anxiety and stress symptoms may be mediated by the changes in RNT. In line with null findings regarding emotional transfer, mediation analyses did not reveal any direct or indirect effects of non-adaptive PASAT performance gains for depression, anxiety and stress symptoms (see Supplemental Material 2). 
the added value of the intervention becomes more compelling. If our data is about as likely under the null hypothesis as it is under the alternative hypothesis however, then the results are inconclusive. All Bayesian analyses were performed using JASP's default Cauchy distribution as a prior.

To properly compare CCT and ACT in terms of emotional transfer, three delta scores per outcome measure were calculated: follow-up (FU) - baseline (PRE), POST - PRE and FU - POST. As such, independent-samples $t$-tests are tests of the (in)difference of the change over time between conditions. Depending on the outcome measures, a successful treatment would be reflected in either decreased scores (DASS, PTQ, CERQ Maladaptive) or increased scores (CERQ Adaptive, Effortful Control) over time. Consequently, the alternative hypothesis, in which we expect the CCT to be more successful than the ACT with regards to the emotional transfer, is dependent on the outcome measure that is tested as well. For instance, we expect that the PTQ delta scores are negative values in both conditions, but to a greater degree (i.e., more negative) in the CCT condition (see Table 4).

All Bayes Factors $\left(\mathrm{BF}_{0 \mathrm{a}}\right)$ for the FU - PRE and the POST - PRE delta scores of the primary outcome measures are 5.088 or higher (see Table 4), which means that the observed delta scores are at least five times more likely under the null hypothesis than under the alternative hypothesis. For the PTQ for example, the data is around seven times more likely under the null hypothesis $\left(\mathrm{BF}_{0 \mathrm{a}} \mathrm{FU}-\right.$ $\mathrm{PRE}=6.75 ; \mathrm{BF}_{0 \mathrm{a}} . \mathrm{POST}-\mathrm{PRE}=7.22$ ). With regards to the $\mathrm{FU}-\mathrm{POST}$ scores of the primary outcome measures, the $\mathrm{BF}_{0 \mathrm{a}}$ tend to be noticeably smaller than for the other delta scores, indicating somewhat less convincing evidence for the null hypothesis when focusing on changes that occurred in the two months after completion of the intervention. Nonetheless, these $\mathrm{BF}_{0 \mathrm{a}}$ systematically point at the data being more likely under the null hypothesis. $\mathrm{The}^{\mathrm{BF}} \mathrm{a}_{0 \mathrm{a}}$ of the secondary outcome measures are less consistent and for some delta scores even inconclusive (see Table 4), making it hard to interpret these results. The delta scores of the Maladaptive items of the CERQ may be an exception though, as the pattern of $\mathrm{BF}_{0 \mathrm{a}}$ seems more in line with the pattern of the primary outcome measures. 


\section{Discussion}

The current study set out to investigate the added value of CCT, operationalized using the adaptive PASAT, combined with a group CBT program targeting fear of failure in undergraduates, as earlier research suggests that CCT may have positive effects on RNT (Hoorelbeke et al., 2016; Hoorelbeke et al., 2015). Currently, the literature on CCT as treatment augmentation, especially in anxious samples, is limited (Course-Choi et al., 2017). On top of the promising findings in the context of depression (Koster et al., 2017), CCT also holds potential from an implementation point-of-view, as it could be incorporated into existing programs through a dedicated online platform, that has been developed taking into account the needs of patients and clinicians (Vervaeke et al., 2018). However, our results suggest that the contribution of CCT over ACT in an anxious sample is limited when combined with a fear of failure training. We will discuss these findings below.

Our analyses show that the participants' performance on the training tasks improves over sessions, followed by significantly better post-intervention scores on the non-adaptive PASAT as compared to baseline, especially in the CCT condition. However, combining fear of failure training with ten 15-minute sessions of CCT (i.e., the adaptive PASAT) does not decrease RNT and symptoms of depression, anxiety and stress more, when compared to the fear of failure training + ACT condition (i.e., the adaptive speed-of-response task). Crucially, the Bayes Factors associated with these analyses suggest that the data is more likely under the null hypothesis than under the alternative hypothesis, indicating that the hypothesized effect of CCT may in fact be absent in our sample.

The explanation for this absence, however, is less clear. We will consider four possibilities in turn: (1) no improvements in cognitive control capabilities following CCT; (2) no impact of CCT on anxiety; (3) similar impact of CCT vs. ACT; (4) ceiling effects due to CBT. First, it cannot be ruled out that the pre to post improvements on the standardized PASAT are largely or even completely explained by the fact that the participants simply improved on their corresponding training tasks, which is rather similar (ACT) or almost identical (CCT) to the standardized PASAT. Put differently, it is possible that there were no specific cognitive improvements on top of task-specific learning, that would have been observed using a different task. Previous research on the adaptive PASAT has shown 
near transfer to the dual n-back task (Hoorelbeke et al., 2016), though not consistently (Vervaeke et al., 2020). As such, future studies in clinical samples should attempt to include additional tasks to establish cognitive transfer effects (cf. Shipstead, Reddick, \& Engle, 2010), if the design allows for such an expansion, without putting too much of a burden on the participants.

Second, it is possible that the adaptive PASAT is simply not effective as an add-on intervention in the context of anxiety. If that is the case, then the additional cognitive load inherent to the adaptive PASAT may not have any impact above and beyond exercising sustained attention, as was required in the active control condition. On the one hand, this is not at odds with existing literature, as beneficial effects have mostly been found in the context of depression (Koster et al., 2017). On the other hand, comorbidity between depression and anxiety is high (Spinhoven et al., 2011). Moreover, RNT is both a key transdiagnostic process (Ehring \& Watkins, 2008) as well as the hypothesized mechanism of CCT (Koster et al., 2017).

Third, the ACT condition may have been effective in reducing RNT as well. Despite the fact that the ACT requires less cognitive processing than the $\mathrm{CCT}$, participants in the control condition still have to put effort into sustaining their attention, in the face of stress, frustration or even negative thoughts and anxiety. Given the nature of our sample, it is possible that repeatedly carrying out a speed-of-response task (ACT) is in fact an elaborate attention exercise, not unlike Wells' Attention Training Technique (Wells, 2000), that has been proven effective even without the extra cognitive load of the adaptive PASAT. The emotional context that the training task provides, is thought to be crucial for the beneficial effects that have been found earlier (Van den Bergh et al., 2018), but may have made the experience of performing the ACT or the CCT too similar in this particular sample. Still, an active control condition is necessary in order to have a stringent test of the experimental treatment, as a waiting list or a more 'passive' control task, insofar such a task exists, may artificially inflate the effectiveness of CCT, due to stronger placebo effects in the experimental condition (Shipstead et al., 2012). The addition of a third condition, in which participants only go to the group sessions, could be a solution here. Ideally, participants in such a third condition would be asked to carry out the online training sessions after completion of the group program and the post-measures, as 
removing the online training tasks (CCT or ACT) entirely could lead to less positive expectancies in this condition as compared to the CCT and ACT.

A fourth potential explanation for the similar outcomes between the CCT and ACT conditions is related to the nature of our research question. The group program, combined with the experience of repeatedly carrying out one of two cognitive tasks, may leave too little room to find an augmentation effect of the adaptive PASAT (as compared to the adaptive speed-of-response task). That this, the effectiveness of the group therapy where RNT is also targeted may have left little room for additive effects of CCT. Again, a third condition that is limited to the group program only would provide insight in this regard.

We believe that the above null findings are an important addition to the literature. E-health interventions such as CCT have large potential to be used as add-on interventions to existing therapies (Van den Bergh et al., 2018). However, establishing the efficacy of such additive interventions is crucial, which also entails transparency about which combinations do not enhance or perhaps even diminish therapy effects. At the theoretical level, this type of null findings can help reflecting about the putative mechanisms that have (or do not have) a causal effect on symptoms. Other notable strengths of the current study are the high level of standardization (at the level of communication, psycho-education and treatment delivery) via the online platform coupled with a naturalistic context for the group program, the respectable participant retention given the nature of the sample (e.g., the tendency to procrastinate) and the use of Bayesian methods for the data analysis. The lack of a fartransfer task and the absence of a third non-augmented condition are limitations however, that should be improved upon in further research. Another avenue for future investigation is the question of moderating variables, as it is unlikely that CCT as a clinical strategy, whether in the context of depression, anxiety or the co-occurrence of both, is (in)effective for all patients. Even within the realm of anxiety, there is a vast amount of heterogeneity, implying that the absence of an effect in one population (i.e., students fearing failure) does not necessarily mean that there cannot be an effect in another (e.g., patients with generalized anxiety disorder). 


\section{Conclusion}

Despite promising results in the context of depression, the current data does not show an added value of Cognitive Control Training (CCT) as treatment augmentation in a sample following a group CBT program targeted at fear of failure. In contrast to our hypotheses, participants randomized to the CCT condition did not show more pronounced reductions in repetitive negative thinking, nor in symptoms related to depression, anxiety and stress, when compared to participants who carried out the active control training instead. This is one of the first studies investigating the use of CCT as an add on intervention in anxiety.

\section{Declaration of Conflicting Interests}

The authors declared that there were no conflicts of interest with respect to the authorship or the publication of this article.

\section{Acknowledgements}

Nathan Van den Bergh is supported by the Concerted Research Action Grant of Ghent University (Grant BOF16/GOA/017), awarded to Rudi De Raedt and Ernst H. W. Koster.

Kristof Hoorelbeke, Jasmien Vervaeke and Ernst H. W. Koster are also supported by an Applied Biomedical (TBM) grant of the Agency for Innovation through Science and Technology (IWT), part of the Research Foundation-Flanders (FWO), PrevenD project (B/14730/01). Kristof Hoorelbeke is a Postdoctoral Fellow of the FWO (FWO.3EO.2018.0031.01).

The authors also thank the participants, the collaborating therapists and Isabelle Lanszweert, head of the student counseling office, without whom the study would not have been possible. 


\section{References}

Anderson, M. C., Bunce, J. G., \& Barbas, H. (2016). Prefrontal-hippocampal pathways underlying inhibitory control over memory. Neurobiology of learning and memory, 134, 145-161. https://doi.org/10.1016/j.nlm.2015.11.008

Antony, M. M., Bieling, P. J., Cox, B. J., Enns, M. W., \& Swinson, R. P. (1998). Psychometric properties of the 42-item and 21-item versions of the Depression Anxiety Stress Scales in clinical groups and a community sample. Psychological assessment, 10(2), 176-181. https://doi.org/10.1037/1040-3590.10.2.176

Auerbach, R. P., Mortier, P., Bruffaerts, R., Alonso, J., Benjet, C., Cuijpers, P., Demyttenaere, K., Ebert, D. D., Green, J. G., \& Hasking, P. (2018). WHO World Mental Health Surveys International College Student Project: prevalence and distribution of mental disorders. Journal of abnormal psychology, 127(7), 623. https://doi.org/10.1037/abn0000362

Cohen, J. D. (2017). Cognitive control: core constructs and current considerations. In The Wiley handbook of cognitive control (pp. 1-28). https://doi.org/10.1002/9781118920497.ch1

Cohen, N., Mor, N., \& Henik, A. (2015). Linking executive control and emotional response: A training procedure to reduce rumination. Clinical Psychological Science, 3(1), 15-25. https://doi.org/10.1177/2167702614530114

Cohen, N., Margulies, D. S., Ashkenazi, S., Schäfer, A., Taubert, M., Henik, A., ... \& Okon-Singer, H. (2016). Using executive control training to suppress amygdala reactivity to aversive information. Neuroimage, 125, 1022-1031. https://doi.org/10.1016/j.neuroimage.2015.10.069

Course-Choi, J., Saville, H., \& Derakshan, N. (2017). The effects of adaptive working memory training and mindfulness meditation training on processing efficiency and worry in high worriers. Behaviour Research and Therapy, 89, 1-13. https://doi.org/10.1016/j.brat.2016.11.002

de Beurs, E., Van Dyck, R., Marquenie, L. A., Lange, A., \& Blonk, R. W. (2001). De DASS: een vragenlijst voor het meten van depressie, angst en stress. Gedragstherapie, 34(1), 35-54. http://www2.psy.unsw.edu.au/Groups/dass/Dutch/DASS-manuscript\%20de\%20Beurs.pdf

De Lissnyder, E., Koster, E. H., Goubert, L., Onraedt, T., Vanderhasselt, M. A., \& De Raedt, R. (2012). Cognitive control moderates the association between stress and rumination. Journal of Behavior Therapy and Experimental Psychiatry, 43(1), 519-525. https://doi.org/10.1016/j.jbtep.2011.07.004

Derryberry, D., \& Rothbart, M. K. (1988). Arousal, affect, and attention as components of temperament. Journal of personality and social psychology, 55(6), 958. https://doi.org/10.1037/0022-3514.55.6.958

Devilly, G. J., \& Borkovec, T. D. (2000). Psychometric properties of the credibility/expectancy questionnaire. Journal of behavior therapy and experimental psychiatry, 31(2), 73-86. https://doi.org/10.1016/S0005-7916(00)00012-4 
Drost, J., Van der Does, W., van Hemert, A. M., Penninx, B. W., \& Spinhoven, P. (2014). Repetitive negative thinking as a transdiagnostic factor in depression and anxiety: A conceptual replication. Behaviour Research and Therapy, 63, 177-183. https://doi.org/10.1016/j.brat.2014.06.004

Ehring, T., Raes, F., Weidacker, K., \& Emmelkamp, P. M. (2012). Validation of the Dutch version of the perseverative thinking questionnaire (PTQ-NL). European Journal of Psychological Assessment. https://doi.org/10.1027/1015-5759/a000097

Ehring, T., \& Watkins, E. R. (2008). Repetitive negative thinking as a transdiagnostic process. International Journal of Cognitive Therapy, 1(3), 192-205. https://doi.org/10.1521/ijct.2008.1.3.192

Ehring, T., Zetsche, U., Weidacker, K., Wahl, K., Schönfeld, S., \& Ehlers, A. (2011). The Perseverative Thinking Questionnaire (PTQ): Validation of a content-independent measure of repetitive negative thinking. Journal of behavior therapy and experimental psychiatry, 42(2), 225-232. https://doi.org/10.1016/j.jbtep.2010.12.003

Eysenck, M. W., Derakshan, N., Santos, R., \& Calvo, M. G. (2007). Anxiety and cognitive performance: attentional control theory. Emotion, 7(2), 336. https://doi.org/10.1037/15283542.7.2.336

Garnefski, N., Kraaij, V., \& Spinhoven, P. (2001). Negative life events, cognitive emotion regulation and emotional problems. Personality and Individual differences, 30(8), 1311-1327. https://doi.org/10.1016/S0191-8869(00)00113-6

Gerwing, T. G., Rash, J. A., Allen Gerwing, A. M., Bramble, B., \& Landine, J. (2015). Perceptions and Incidence of Test Anxiety. Canadian Journal for the Scholarship of Teaching and Learning, 6(3), 3. https://doi.org/10.5206/cjsotl-rcacea.2015.3.3

Godfrin, K., Goeleven, E., \& Schoof, P. (2004). Dutch translation of the Credibility/Expectancy Questionnaire (CEQ-NL).

Goschke, T. (2014). Dysfunctions of decision-making and cognitive control as transdiagnostic mechanisms of mental disorders: advances, gaps, and needs in current research. International journal of methods in psychiatric research, 23(S1), 41-57. https://doi.org/10.1002/mpr.1410

Grahek, I., Everaert, J., Krebs, R. M., \& Koster, E. H. (2018). Cognitive control in depression: Toward clinical models informed by cognitive neuroscience. Clinical Psychological Science, 6(4), 464-480. https://doi.org/10.1177/2167702618758969

Gronwall, D. (1977). Paced auditory serial-addition task: a measure of recovery from concussion. Perceptual and motor skills, 44(2), 367-373. https://doi.org/10.2466/pms.1977.44.2.367

Hankin, B. L., Snyder, H. R., Gulley, L. D., Schweizer, T. H., Bijttebier, P., Nelis, S., Toh, G., \& Vasey, M. W. (2016). Understanding comorbidity among internalizing problems: Integrating 
latent structural models of psychopathology and risk mechanisms. Development and psychopathology, 28(4pt1), 987-1012. https://doi.org/10.1017/S0954579416000663

Hartman, C. A., Majdandžić, M., \& Rothbart, M. K. (2001). Temperament: Nederlandse vertaling $A T Q$. https://research.bowdoin.edu/rothbart-temperament-questionnaires/request-forms/

Henry, J. D., \& Crawford, J. R. (2005). The short-form version of the Depression Anxiety Stress Scales (DASS-21): Construct validity and normative data in a large non-clinical sample. British journal of clinical psychology, 44(2), 227-239. https://doi.org/10.1348/014466505X29657

Hirsch, C. R., \& Mathews, A. (2012). A cognitive model of pathological worry. Behaviour Research and Therapy, 50(10), 636-646. https://doi.org/10.1016/j.brat.2012.06.007

Hoorelbeke, K., \& Koster, E. H. (2017). Internet-delivered cognitive control training as a preventive intervention for remitted depressed patients: Evidence from a double-blind randomized controlled trial study. Journal of consulting and clinical psychology, 85(2), 135. https://doi.org/10.1037/ccp0000128

Hoorelbeke, K., Koster, E. H., Demeyer, I., Loeys, T., \& Vanderhasselt, M.-A. (2016). Effects of cognitive control training on the dynamics of (mal) adaptive emotion regulation in daily life. Emotion, 16(7), 945. https://doi.org/10.1037/emo0000169

Hoorelbeke, K., Koster, E. H., Vanderhasselt, M.-A., Callewaert, S., \& Demeyer, I. (2015). The influence of cognitive control training on stress reactivity and rumination in response to a lab stressor and naturalistic stress. Behaviour Research and Therapy, 69, 1-10. https://doi.org/10.1016/j.brat.2015.03.010

Joormann, J., \& D'Avanzato, C. (2010). Emotion regulation in depression: Examining the role of cognitive processes: Cognition \& Emotion Lecture at the 2009 ISRE Meeting. Cognition and Emotion, 24(6), 913-939. https://doi.org/10.1080/02699931003784939

Klemanski, D. H., Curtiss, J., McLaughlin, K. A., \& Nolen-Hoeksema, S. (2017). Emotion regulation and the transdiagnostic role of repetitive negative thinking in adolescents with social anxiety and depression. Cognitive therapy and research, 41(2), 206-219. https://doi.org/10.1007/s10608-016-9817-6

Koster, E. H., De Lissnyder, E., Derakshan, N., \& De Raedt, R. (2011). Understanding depressive rumination from a cognitive science perspective: The impaired disengagement hypothesis. Clinical psychology review, 31(1), 138-145. https://doi.org/10.1016/j.cpr.2010.08.005

Koster, E. H., Hoorelbeke, K., Onraedt, T., Owens, M., \& Derakshan, N. (2017). Cognitive control interventions for depression: A systematic review of findings from training studies. Clinical psychology review, 53, 79-92. https://doi.org/10.1016/j.cpr.2017.02.002

Kruschke, J. K. (2011). Introduction to special section on Bayesian data analysis. Perspectives on Psychological Science, 6(3), 272. https://doi.org/10.1177/1745691611406926 
Lakens, D. (2013). Calculating and reporting effect sizes to facilitate cumulative science: a practical primer for t-tests and ANOVAs. Frontiers in psychology, 4, 863. https://doi.org/10.3389/fpsyg.2013.00863

Lovibond, P. F., \& Lovibond, S. H. (1995). The structure of negative emotional states: Comparison of the Depression Anxiety Stress Scales (DASS) with the Beck Depression and Anxiety Inventories. Behaviour Research and Therapy, 33(3), 335-343. https://doi.org/10.1016/00057967(94)00075-U

McEvoy, P. M., Watson, H., Watkins, E. R., \& Nathan, P. (2013). The relationship between worry, rumination, and comorbidity: Evidence for repetitive negative thinking as a transdiagnostic construct. Journal of affective disorders, 151(1), 313-320. https://doi.org/10.1016/j.jad.2013.06.014

Miller, E. K., \& Cohen, J. D. (2001). An integrative theory of prefrontal cortex function. Annual review of neuroscience, 24(1), 167-202. https://doi.org/10.1146/annurev.neuro.24.1.167

Miyake, A., Friedman, N. P., Emerson, M. J., Witzki, A. H., Howerter, A., \& Wager, T. D. (2000). The unity and diversity of executive functions and their contributions to complex "frontal lobe" tasks: A latent variable analysis. Cognitive psychology, 41(1), 49-100. https://doi.org/10.1006/cogp.1999.0734

Moshier, S. J., \& Otto, M. W. (2017). Behavioral activation treatment for major depression: A randomized trial of the efficacy of augmentation with cognitive control training. Journal of affective disorders, 210, 265-268. https://doi.org/10.1016/j.jad.2017.01.003

Mulder, J., \& Wagenmakers, E.-J. (2016). Editors' introduction to the special issue "Bayes factors for testing hypotheses in psychological research: Practical relevance and new developments". Journal of Mathematical Psychology, 72, 1-5. https://doi.org/10.1016/j.jmp.2016.01.002

Onraedt, T., \& Koster, E. H. (2014). Training working memory to reduce rumination. PloS one, 9(3). https://doi.org/10.1371/journal.pone.0090632

Osman, A., Wong, J. L., Bagge, C. L., Freedenthal, S., Gutierrez, P. M., \& Lozano, G. (2012). The depression anxiety stress Scales - 21 (DASS-21): further examination of dimensions, scale reliability, and correlates. Journal of clinical psychology, 68(12), 1322-1338. https://doi.org/10.1002/jclp.21908

Owens, M., Koster, E. H., \& Derakshan, N. (2013). Improving attention control in dysphoria through cognitive training: Transfer effects on working memory capacity and filtering efficiency. Psychophysiology, 50(3), 297-307. https://doi.org/10.1111/psyp.12010

Philippot, P., \& Brutoux, F. (2008). Induced rumination dampens executive processes in dysphoric young adults. Journal of behavior therapy and experimental psychiatry, 39(3), 219-227. https://doi.org/10.1016/j.jbtep.2007.07.001 
Sari, B. A., Koster, E. H., \& Derakshan, N. (2017). The effects of active worrying on working memory capacity. Cognition and Emotion, 31(5), 995-1003. https://doi.org/10.1080/02699931.2016.1170668

Shi, R., Sharpe, L., \& Abbott, M. (2019). A meta-analysis of the relationship between anxiety and attentional control. Clinical psychology review, 101754. https://doi.org/10.1016/j.cpr.2019.101754

Shipstead, Z., Hicks, K. L., \& Engle, R. W. (2012). Working memory training remains a work in progress. https://doi.org/10.1016/j.jarmac.2012.07.009

Shipstead, Z., Lindsey, D. R., Marshall, R. L., \& Engle, R. W. (2014). The mechanisms of working memory capacity: Primary memory, secondary memory, and attention control. Journal of Memory and Language, 72, 116-141. https://doi.org/10.1016/j.jml.2014.01.004

Shipstead, Z., Redick, T. S., \& Engle, R. W. (2010). Does working memory training generalize?. Psychologica Belgica, 50(3), 245-276. http://doi.org/10.5334/pb-50-3-4-245

Siegle, G. J., Ghinassi, F., \& Thase, M. E. (2007). Neurobehavioral therapies in the 21st century: Summary of an emerging field and an extended example of cognitive control training for depression. Cognitive therapy and research, 31(2), 235-262. https://doi.org/10.1007/s10608006-9118-6

Siegle, G. J., Price, R. B., Jones, N. P., Ghinassi, F., Painter, T., \& Thase, M. E. (2014). You gotta work at it: Pupillary indices of task focus are prognostic for response to a neurocognitive intervention for rumination in depression. Clinical Psychological Science, 2(4), 455-471. https://doi.org/10.1177/2167702614536160

Snyder, H. R., \& Hankin, B. L. (2016). Spiraling out of control: Stress generation and subsequent rumination mediate the link between poorer cognitive control and internalizing psychopathology. Clinical Psychological Science, 4(6), 1047-1064. https://doi.org/10.1177/2167702616633157

Snyder, H. R., Miyake, A., \& Hankin, B. L. (2015). Advancing understanding of executive function impairments and psychopathology: bridging the gap between clinical and cognitive approaches. Frontiers in psychology, 6, 328. https://doi.org/10.3389/fpsyg.2015.00328

Spinhoven, P., Drost, J., van Hemert, B., \& Penninx, B. W. (2015). Common rather than unique aspects of repetitive negative thinking are related to depressive and anxiety disorders and symptoms. Journal of Anxiety Disorders, 33, 45-52. https://doi.org/10.1016/j.janxdis.2015.05.001

Spinhoven, P., van Balkom, A., \& Nolen, W. A. (2011). Comorbidity patterns of anxiety and depressive disorders in a large cohort study: the Netherlands Study of Depression and Anxiety (NESDA). Journal of Clinical Psychiatry, 72, 341-348.

https://doi.org/10.4088/JCP.10m06176blu 
Schweizer, S., Grahn, J., Hampshire, A., Mobbs, D., \& Dalgleish, T. (2013). Training the emotional brain: improving affective control through emotional working memory training. Journal of Neuroscience, 33(12), 5301-5311. https://doi.org/10.1523/JNEUROSCI.2593-12.2013

Van den Bergh, N., Hoorelbeke, K., De Raedt, R., \& Koster, E. H. (2018). Remediation of depressionrelated cognitive impairment: cognitive control training as treatment augmentation. Expert review of neurotherapeutics, 18(12), 907-913.

https://doi.org/10.1080/14737175.2018.1537783

Vanderhasselt, M.-A., Koster, E. H., Onraedt, T., Bruyneel, L., Goubert, L., \& De Raedt, R. (2014). Adaptive cognitive emotion regulation moderates the relationship between dysfunctional attitudes and depressive symptoms during a stressful life period: A prospective study. Journal of behavior therapy and experimental psychiatry, 45(2), 291-296.

https://doi.org/10.1016/j.jbtep.2014.01.003

Vervaeke, J., Hoorelbeke, K., Baeken, C., Van Looy, J., \& Koster, E. H. (2020). Transfer and motivation after cognitive control training for remitted depression in healthy sample. Journal of Cognitive Enhancement, 4, 49-61. https://doi.org/10.1007/s41465-019-00135-6

Vervaeke, J., Van Looy, J., Hoorelbeke, K., Baeken, C., \& Koster, E. H. (2018). Gamified cognitive control training for remitted depressed individuals: user requirements analysis. JMIR serious games, 6(2), e6. https://doi.org/10.2196/games.8609

Wanmaker, S., Geraerts, E., \& Franken, I. H. (2015). A working memory training to decrease rumination in depressed and anxious individuals: A double-blind randomized controlled trial. Journal of affective disorders, 175, 310-319. https://doi.org/10.1016/j.jad.2014.12.027

Watkins, E., \& Brown, R. (2002). Rumination and executive function in depression: An experimental study. Journal of Neurology, Neurosurgery \& Psychiatry, 72(3), 400-402. https://doi.org/10.1136/jnnp.72.3.400

Wells, A. (2000). New pathways for cognitive restructuring: attention modifications (ATT and SAR). In Emotional Disorders and Meta-Cognition. New York: Wiley (pp. 132-154). https://doi.org/10.1002/9780470713662.ch9

Zeidner, M., \& Mathews, G. (2005). Evaluation anxiety. In A. J. Elliot \& C. S. Dweck (Eds.), Handbook of competence and motivation (pp.141-163). London: Guildford Press.

Zetsche, U., Bürkner, P.-C., \& Schulze, L. (2018). Shedding light on the association between repetitive negative thinking and deficits in cognitive control-A meta-analysis. Clinical psychology review, 63, 56-65. https://doi.org/10.1016/j.cpr.2018.06.001 
Figures \& Tables

Figure 1. CONSORT flow diagram.

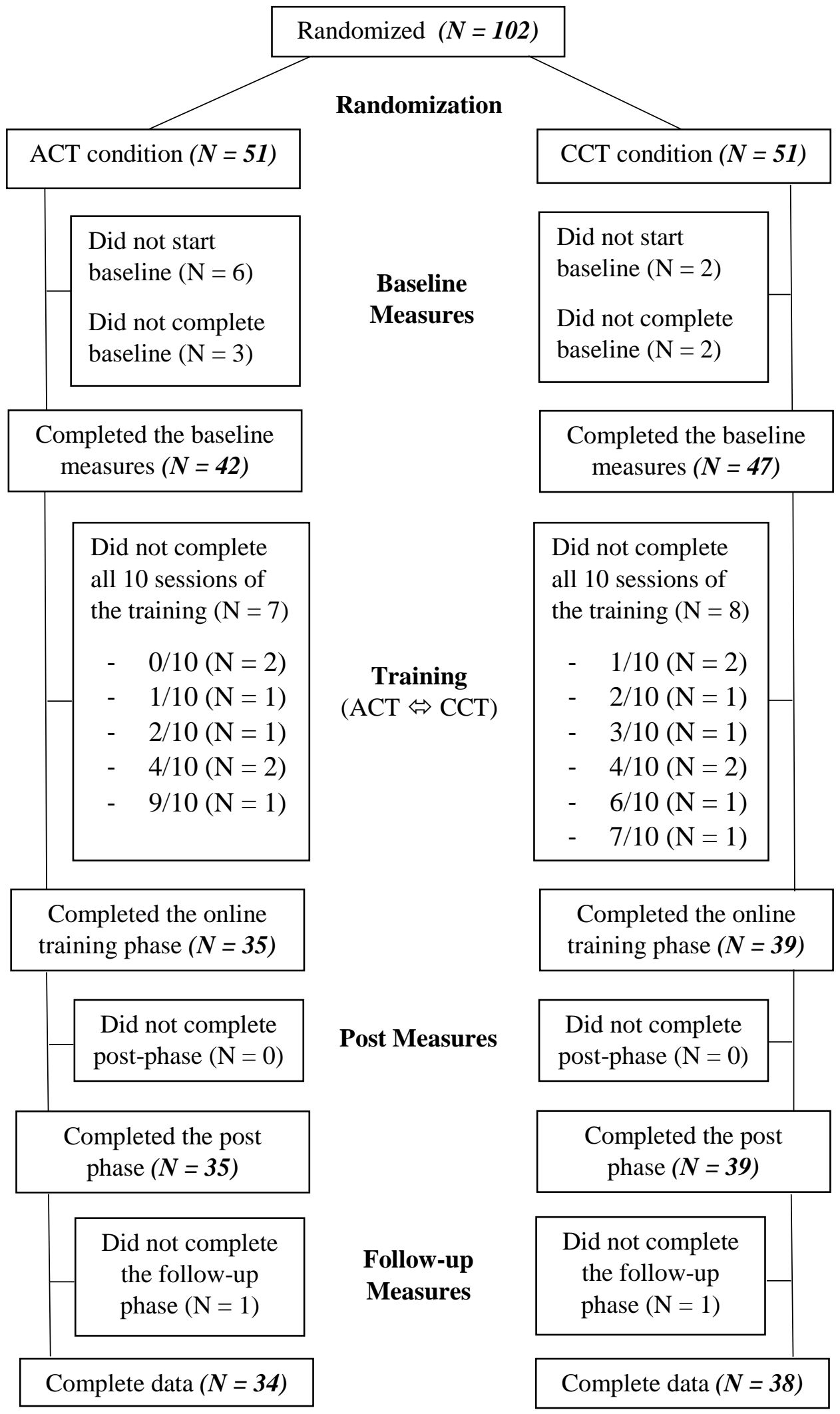




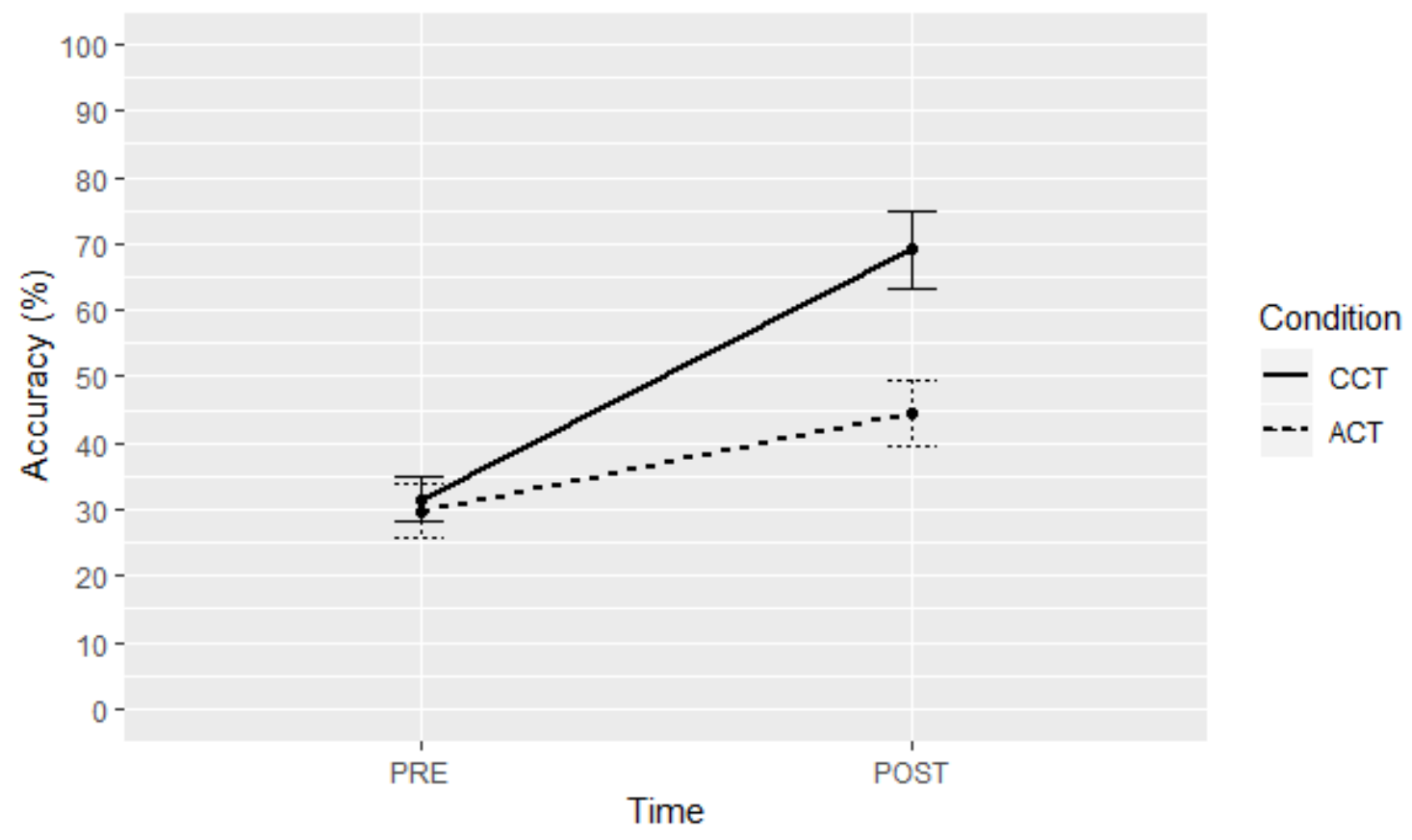

Figure 2. Performance on the non-adaptive Paced Auditory Serial Addition Task (PASAT), in terms of accuracy, as a function of time and group (bars represent $95 \%$ confidence intervals). PRE = baseline; POST = post-intervention; $\mathrm{CCT}=$ Cognitive Control Training; $\mathrm{ACT}=$ Active Control Training 
Table 1

Information regarding demographics and study progress, by condition

\begin{tabular}{|c|c|c|c|c|c|}
\hline & $\mathrm{ACT}^{1}(\mathrm{~N}=42)$ & $\mathrm{CCT}^{1}(\mathrm{~N}=47)$ & Statistic & df & $p$ \\
\hline Gender (female:male) & $35: 7$ & $36: 11$ & $\chi^{2}=0.62$ & 1 & .430 \\
\hline Age (SD) & $20.5(2.3)$ & $21.0(2.3)$ & $t=1.17$ & 86.2 & .244 \\
\hline Current study level Bachelor:Master ${ }^{2}$ & $36: 6$ & $35: 11$ & $\chi^{2}=1.31$ & 1 & .253 \\
\hline History of mental health medication use no:yes & $32: 10$ & $40: 7$ & $\chi^{2}=1.14$ & 1 & .285 \\
\hline History of therapeutic contact no:yes & $17: 25$ & $16: 31$ & $\chi^{2}=0.39$ & 1 & .531 \\
\hline History telephone support/crisis lines no:yes & $40: 2$ & $46: 1$ & $O R=2.28$ & --- & .600 \\
\hline History of self-help (internet, book,...) no:yes & $33: 9$ & $30: 17$ & $\chi^{2}=2.33$ & 1 & .127 \\
\hline Group program sessions missed no:yes:unknown ${ }^{3}$ & 19:16:7 & $23: 16: 8$ & $\chi^{2}=0.17$ & 2 & .920 \\
\hline Days from baseline to training (SD) ${ }^{4}$ & $1.5(1.3)$ & $2.0(1.9)$ & $t=1.40$ & 81.6 & .165 \\
\hline Days spent to carry out the training (SD) ${ }^{4}$ & $11.6(3.3)$ & $12.0(3.8)$ & $t=0.47$ & 71.9 & .643 \\
\hline Days from training to post-assessment (SD) ${ }^{4}$ & $13.8(9.0)$ & $12.2(8.1)$ & $t=-0.84$ & 68.7 & .405 \\
\hline Days from training to follow-up (SD) ${ }^{4}$ & $73.4(8.7)$ & $72.7(9.6)$ & $t=-0.32$ & 70.0 & .746 \\
\hline
\end{tabular}

${ }^{1} \mathrm{ACT}$ and CCT refer to the Active Control Training and Cognitive Control Training conditions, respectively.

${ }^{2}$ There was one PhD student in the sample (in the CCT condition), who was not taken into account for the analysis comparing the students' level.

${ }^{3}$ If participants missed group sessions, they missed either one ( $\mathrm{N}=12$ in ACT, $\mathrm{N}=14$ in CCT) or two ( $\mathrm{N}=4$ in ACT, $\mathrm{N}=2$ in CCT) sessions.

${ }^{4}$ Means, standard deviations and $t$-tests are based on the available data (i.e., not taking into account the participants who dropped out at any stage in the study:

after the baseline measures but before the first online session, during the online training phase or between post and follow-up phases. 
Table 2

Mean scores on questionnaires and tasks, divided by group and assessment, with standard deviations in parentheses

\section{Active Control Training $(N=42)$}

Cognitive Control Training $(N=47)$

\begin{tabular}{|c|c|c|c|c|c|c|}
\hline & PRE & POST & FU & PRE & POST & $\mathrm{FU}$ \\
\hline \multicolumn{7}{|c|}{ Credibility/expectancy } \\
\hline CEQ Credibility & $0.1(2.3)$ & $-0.1(2.8)$ & --- & $-0.1(2.6)$ & $-0.4(2.8)$ & --- \\
\hline CEQ Expectancy & $0.3(2.6)$ & $0.3(2.7)$ & --- & $-0.2(2.4)$ & $-0.4(2.8)$ & --- \\
\hline \multicolumn{7}{|c|}{ Primary outcome measures } \\
\hline DASS Depression & $8.8(5.2)$ & $5.8(5.1)$ & $5.8(4.9)$ & $7.8(5.3)$ & $6.1(5.5)$ & $5.9(5.0)$ \\
\hline DASS Anxiety & $7.9(4.8)$ & $6.0(3.9)$ & $5.3(3.9)$ & $7.3(4.8)$ & $5.5(4.7)$ & $5.0(4.5)$ \\
\hline DASS Stress & $10.3(4.5)$ & $7.8(4.5)$ & $8.1(4.2)$ & $9.3(5.1)$ & $7.7(4.8)$ & $7.4(4.5)$ \\
\hline PTQ (total score) & $42.6(9.9)$ & $37.1(9.8)$ & $35.3(9.8)$ & $40.1(9.3)$ & $35.9(10.1)$ & $33.9(9.9)$ \\
\hline \multicolumn{7}{|c|}{ Secondary outcome measures } \\
\hline CERQ Adaptive & $54.0(10.8)$ & $58.2(11.3)$ & $57.1(10.0)$ & $54.2(14.3)$ & $57.0(12.8)$ & $58.7(13.5)$ \\
\hline CERQ Maladaptive & $42.7(8.1)$ & $39.1(7.6)$ & $38.6(6.7)$ & $42.2(9.8)$ & $39.1(9.9)$ & $38.2(9.0)$ \\
\hline ATQ Effortful Control & $3.8(0.8)$ & $3.9(0.8)$ & $4.0(0.7)$ & $4.1(0.8)$ & $4.4(0.7)$ & $4.5(0.7)$ \\
\hline \multicolumn{7}{|c|}{ Task-specific cognitive transfer } \\
\hline PASAT acc. $(\%)$ & $29.7(12.9)$ & $44.4(15.8)$ & --- & $31.6(11.3)$ & $69.1(20.3)$ & --- \\
\hline
\end{tabular}

PRE = baseline; POST = post-intervention; FU = follow-up; CEQ = Credibility/Expectancy Questionnaire; DASS = Depression Anxiety Stress Scales; PTQ = Perseverative Thinking Questionnaire; CERQ = Cognitive Emotion Regulation Questionnaire; ATQ Effortful Control = Effortful Control factor from the Adult Temperament Questionnaire; PASAT $=$ non-adaptive Paced Auditory Serial Addition Task; acc. $(\%)=$ accuracy (as percentage). 
Table 3

Repeated Measures ANOVAs

\begin{tabular}{|c|c|c|c|c|c|c|c|c|c|c|c|c|}
\hline & \multicolumn{4}{|c|}{ Main effect of Time } & \multicolumn{4}{|c|}{ Main effect of Group } & \multicolumn{4}{|c|}{ Time x Group interaction } \\
\hline & $F$ & $f$ & $p$ & $p^{2}$ & $F$ & $d f$ & $p$ & $p^{2}$ & $F$ & $d f$ & $p$ & $\eta p^{2}$ \\
\hline \multicolumn{13}{|c|}{ Credibility/expectancy } \\
\hline CEQ Credibility & 0.72 & 1,87 & .397 & .01 & 0.13 & 1,87 & .718 & $<.01$ & 0.03 & 1,87 & .864 & $<.01$ \\
\hline CEQ Expectancy & 0.03 & 1,87 & .859 & $<.01$ & 1.50 & 1,87 & .223 & .02 & 0.23 & 1,87 & .631 & $<.01$ \\
\hline \multicolumn{13}{|c|}{ Primary outcome measures } \\
\hline DASS Depression & 18.36 & 2,174 & $<.001$ & .17 & 0.04 & 1,87 & .838 & $<.01$ & 1.25 & 2,174 & .290 & .01 \\
\hline DASS Anxiety & 18.14 & 2,174 & $<.001$ & .17 & 0.30 & 1,87 & .587 & $<.01$ & 0.08 & 2,174 & .921 & $<.01$ \\
\hline DASS Stress & 14.45 & 2,174 & $<.001$ & .14 & 0.46 & 1,87 & .500 & .01 & 0.46 & 2,174 & .634 & .01 \\
\hline PTQ (total score) & 39.72 & 2,174 & $<.001$ & .31 & 0.86 & 1,87 & .357 & .01 & 0.36 & 2,174 & $.700 *$ & $<.01$ \\
\hline \multicolumn{13}{|c|}{ Secondary outcome measures } \\
\hline CERQ Adaptive & 9.49 & 2,174 & $<.001$ & .10 & 0.01 & 1,87 & .930 & $<.01$ & 0.99 & 2,174 & $.375^{*}$ & .01 \\
\hline CERQ Maladaptive & 25.83 & 2,174 & $<.001$ & .23 & 0.03 & 1,87 & .857 & $<.01$ & 0.08 & 2,174 & .926 & $<.01$ \\
\hline ATQ Effortful Control & 24.99 & 2,174 & $<.001$ & .22 & 7.98 & 1,87 & $<.01$ & .08 & 0.78 & 2,174 & .461 & .01 \\
\hline \multicolumn{13}{|c|}{ Task-specific cognitive transfer } \\
\hline PASAT acc. $(\%)$ & 229.38 & 1,87 & $<.001$ & .73 & 22.26 & 1,87 & $<.001$ & .20 & 44.18 & 1,87 & $<.001$ & .34 \\
\hline
\end{tabular}

$\overline{\mathrm{CEQ}}=$ Credibility/Expectancy Questionnaire; DASS = Depression Anxiety Stress Scales; PTQ = Perseverative Thinking Questionnaire; CERQ = Cognitive Emotion Regulation Questionnaire; ATQ Effortful Control = Effortful Control factor from the Adult Temperament Questionnaire; PASAT = non-adaptive Paced Auditory Serial Addition Task; acc. $(\%)=$ accuracy (as percentage).

* Mauchly's test of sphericity indicated that the assumption of sphericity was violated $(p<.05)$ for the (total) PTQ score and the adaptive compound score of the CERQ. However, even when using the most stringent sphericity correction (i.e., Greenhouse-Geisser), the results remain the same. Only the estimated $p$-values of the Time x Group interaction differ slightly: .685 instead of .700 (CERQ Adaptive) and .368 instead of .375 (PTQ). 


\section{Table 4}

Post-hoc Bayesian independent-samples t-tests of primary and secondary outcome measures (delta scores)

\section{$\mathbf{B F}_{0 a}\left(\mathbf{B F}_{\mathbf{a} 0}\right)$}

\begin{tabular}{lcccc}
\hline & FU - PRE & POST - PRE & FU - POST & Alternative Hypothesis \\
\hline PASAT acc. (\%) & -- & $\begin{array}{c}1.411 \times 10^{-7} \\
\left(7.090 \times 10^{6}\right)\end{array}$ & --- & Cognitive Control Training > Active Control Training \\
\multicolumn{1}{c}{ Primary outcome measures } & & & \\
DASS Depression & $8.969(0.111)$ & $10.780(0.093)$ & $3.774(0.265)$ & Cognitive Control Training < Active Control Training \\
DASS Anxiety & $5.895(0.170)$ & $5.088(0.197)$ & $5.325(0.188)$ & Cognitive Control Training < Active Control Training \\
DASS Stress & $5.500(0.182)$ & $8.178(0.122)$ & $2.649(0.378)$ & Cognitive Control Training < Active Control Training \\
PTQ (total score) & $6.745(0.148)$ & $7.223(0.138)$ & $3.993(0.250)$ & Cognitive Control Training < Active Control Training \\
\multicolumn{1}{c}{ Secondary outcome measures } & & & \\
CERQ Adaptive & $2.642(0.378)$ & $7.167(0.140)$ & $0.793(1.262)$ & Cognitive Control Training > Active Control Training \\
CERQ Maladaptive & $4.698(0.213)$ & $5.744(0.174)$ & $3.389(0.295)$ & Cognitive Control Training < Active Control Training \\
ATQ Effortful Control & $2.098(0.477)$ & $1.466(0.682)$ & $5.758(0.174)$ & Cognitive Control Training > Active Control Training
\end{tabular}

$\mathrm{BF}_{0 \mathrm{a}}\left(\mathrm{BF}_{\mathrm{a} 0}\right)=$ Bayes Factors of the null vs the alternative hypothesis (Bayes Factors of alternative vs null hypothesis); PRE = baseline; POST = post-intervention; FU = follow-up. 


\section{Supplemental Material 1: CEQ}

The table (S1) below shows the means and standard deviations for each of the specific CEQ items, per condition and per time point. Overall, the item means are between 4.5 and 6.5 out of 9 , which suggests moderate levels of credibility and (positive) expectancy at best. The means of items that ask about expected percentage of symptom reduction (both in terms of thinking and feeling) are relatively high, given that the items were specifically referring to the Cognitive Control Training only (i.e., around 40\%). There is a high amount of variability however, implying that these general trends should be interpreted carefully. In line with the standardized total scores (cf. table 2), most credibility and expectancy items seem to be scored slightly lower after training was completed, though not significantly (cf. table 3 ).

Table S1

Means and standard deviations of CEQ items, per condition and per time point

$\operatorname{ACT}(\mathrm{N}=42) \quad \operatorname{CCT}(\mathrm{N}=47)$

$\begin{array}{llll}\text { PRE } & \text { POST } & \text { PRE } & \text { POST }\end{array}$

\section{Credibility}

How logical is the treatment? (9-point scale)

$6.5(1.3) \quad 6.0(1.6) \quad 6.3(1.5) \quad 5.9(1.5)$

How successful in reducing symptoms? (9-point scale)

$5.5(1.2) \quad 5.0(1.8) \quad 5.6(1.3) \quad 5.0(1.6)$

How confident in recommending? (9-point scale)

$5.8(1.5) \quad 5.1(2.0) \quad 5.8(1.9)$

$4.8(2.2)$

\section{Expectancy}

\begin{tabular}{lllll} 
Expected symptom improvement: thinking (\% reduced) & $40.5(18.6)$ & $37.4(19.9)$ & $41.9(19.2)$ & $34.0(21.6)$ \\
How much do you feel that treatment will help? (9-point scale) & $5.5(1.5)$ & $5.2(1.9)$ & $4.8(1.5)$ & $4.6(1.7)$ \\
Expected symptom improvement: feeling (\% reduced) & $40.2(23.1)$ & $41.7(22.3)$ & $37.7(20.9)$ & $35.5(24.0)$ \\
\hline CEQ = Credibility/Expectancy Questionnaire & & & &
\end{tabular}




\section{Supplemental Material 2: Mediation Analyses}

We conducted multiple regression analyses to test the hypothesis that Cognitive Control Training (CCT) effects anxiety, depression and stress symptoms, via Repetitive Negative Thinking (RNT). To that end, delta scores of performance on the non-adaptive Paced Auditory Serial Addition Task (PASAT) were calculated (post-intervention accuracy minus baseline accuracy), to quantify the degree of improvement after versus before training. These delta scores are the independent variable $(\mathrm{X})$ in the mediation models. Post-intervention RNT is the mediating variable $(\mathrm{M})$, whereas depression, anxiety and stress symptoms measured at two-months follow-up are the dependent variables (Ys) in models 1, 2 and 3 respectively (see figure S2 below). Baseline RNT as well as baseline symptoms (depression, anxiety or stress, depending on the model) were added as covariates, to control for effects of baseline functioning on the dependent variable.

Analyses were carried out on the completers-only sample $(\mathrm{N}=72$ or $80.9 \%$ of the total sample), due to the nature of the drop-out. Participants dropping out at one point (e.g., during the training phase) do not drop in at a later stage (e.g., for the post-intervention measures). Consequently, carrying forward the last known observations would result in artificially increasing the covariation between the variables in the mediation models. The 95\% Confidence Intervals (CIs) for the indirect effects were obtained via the Preacher and Hayes bootstrapping method (Preacher \& Hayes, 2004, 2008), using 5000 resamples.

The results (see table S2 below) indicate that one hypothesized path is present, in one model only: post-training RNT (the mediating variable) significantly predicted depressive symptoms (the dependent variable of model 1$)$ at two months follow-up $(B=0.15, t=2.38,95 \% C I=[0.02,0.27])$. Put differently, these results suggest that performance increases on the non-adaptive PASAT (postintervention compared to baseline) do not predict depression, anxiety and stress symptoms, indirectly (via RNT) or otherwise. This is in line with our finding that the contribution of CCT (ten 15-minute sessions of the adaptive PASAT) over ACT (ten 15-minute session of an adaptive speed-of-response task) in an anxious sample is limited in terms of depression, anxiety and stress symptoms, when combined with a fear of failure training. 
Figure S2. Visualization of the mediation models, differing only in terms of the dependent variable (and its corresponding baseline covariate).
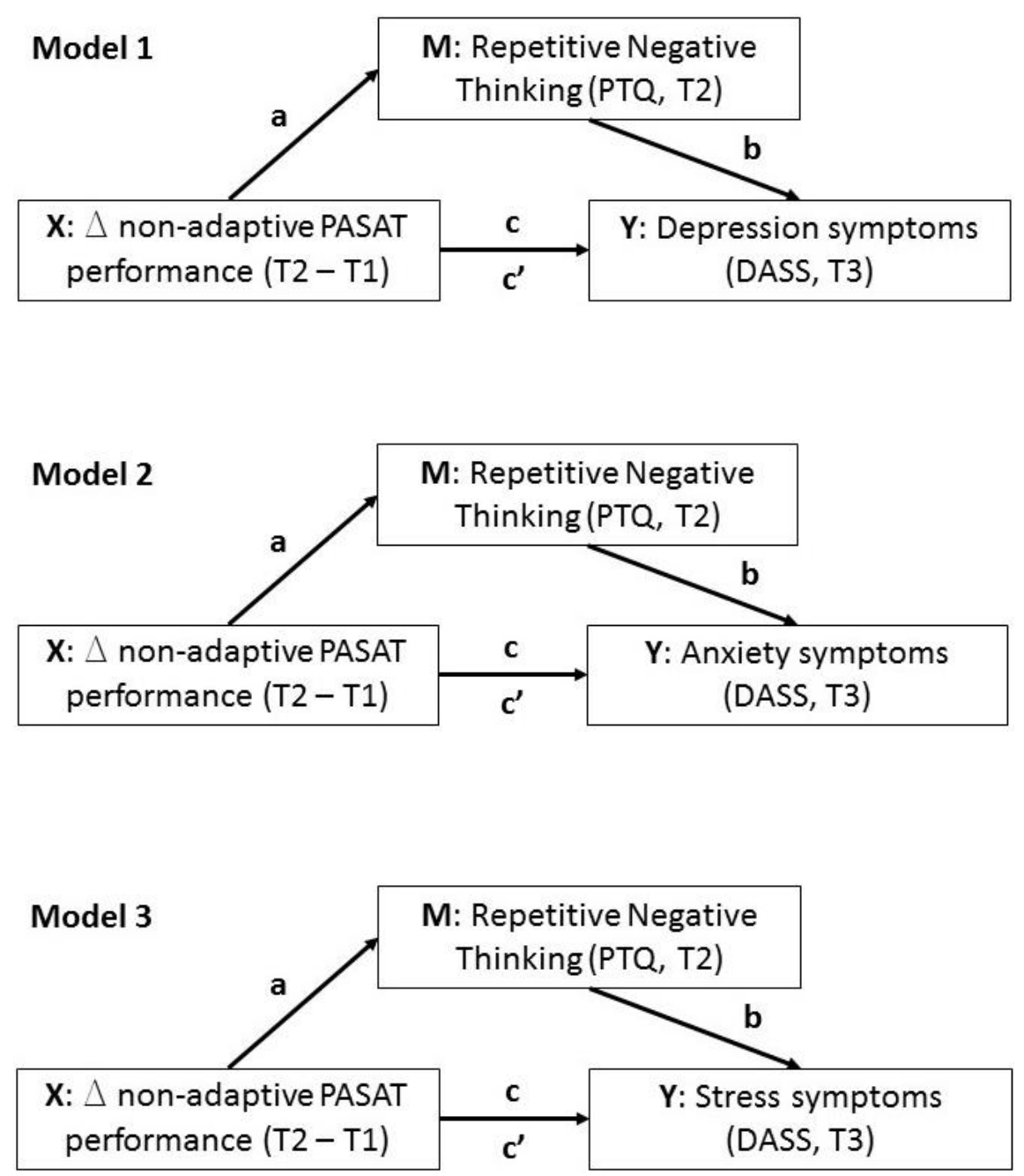

$\mathrm{T} 1=$ baseline $; \mathrm{T} 2$ = post-intervention; $\mathrm{T} 3=$ follow-up DASS $=$ Depression Anxiety Stress Scales; PTQ $=$ Perseverative Thinking Questionnaire; PASAT $=$ Paced Auditory Serial Addition Task 
Overview of mediation analyses' results

\begin{tabular}{|c|c|c|c|c|c|c|c|c|c|}
\hline & \multicolumn{3}{|c|}{ Model 1 (Depression) } & \multicolumn{4}{|c|}{ Model 2 (Anxiety) } & \multicolumn{2}{|c|}{ Model 3 (Stress) } \\
\hline & $B$ & $t$ & $95 \% C I$ & $B$ & $t$ & $95 \% C I$ & $B$ & $t$ & $95 \% C I$ \\
\hline \multicolumn{10}{|l|}{ Model paths } \\
\hline $\mathrm{X} \rightarrow \mathrm{M}(\mathrm{a})$ & 1.08 & 0.20 & {$[-9.59,11.74]$} & 1.13 & 0.21 & {$[-9.55,11.82]$} & 1.02 & 0.19 & {$[-9.73,11.77]$} \\
\hline $\mathrm{M} \rightarrow \mathrm{Y}(\mathrm{b})$ & 0.15 & 2.38 & {$[0.02,0.27]$} & 0.02 & 0.29 & {$[-0.09,0.13]$} & 0.02 & 0.40 & {$[-0.09,0.13]$} \\
\hline $\mathrm{X} \rightarrow \mathrm{Y}$ (total effect, c) & -0.14 & -0.05 & {$[-5.68,5.40]$} & -1.72 & -0.71 & {$[-6.53,3.09]$} & -3.85 & -1.57 & {$[-8.74,1.04]$} \\
\hline$X \rightarrow Y$ (direct effect, c') & -0.30 & -0.11 & {$[-5.66,5.07]$} & -1.74 & -0.72 & {$[-6.58,3.10]$} & -3.87 & -1.57 & {$[-8.79,1.05]$} \\
\hline $\mathrm{X} \rightarrow \mathrm{M} \rightarrow \mathrm{Y}$ (indirect effect) & 0.16 & --- & {$[-1.73,1.74] *$} & 0.02 & --- & {$[-0.51,0.78] *$} & 0.02 & --- & {$[-0.78,0.71] *$} \\
\hline \multicolumn{10}{|l|}{ Covariates } \\
\hline Baseline PTQ (total score) & -0.02 & -0.30 & {$[-0.16,0.12]$} & -0.08 & -1.17 & {$[-0.21,0.05]$} & -0.01 & -0.13 & {$[-0.14,0.12]$} \\
\hline Baseline DASS Depression & 0.37 & 3.45 & {$[0.16,0.59]$} & --- & --- & --- & --- & --- & --- \\
\hline Baseline DASS Anxiety & --- & --- & --- & 0.56 & 5.20 & {$[0.34,0.77]$} & --- & --- & --- \\
\hline Baseline DASS Stress & --- & --- & --- & --- & --- & --- & 0.40 & 3.58 & {$[0.18,0.63]$} \\
\hline
\end{tabular}

* Confidence Interval (CI) obtained via the Preacher and Hayes bootstrapping method (5000 samples).

\section{Additional References}

Preacher, K. J., \& Hayes, A. F. (2004). SPSS and SAS procedures for estimating indirect effects in simple mediation models. Behavior research methods, instruments, \& computers, 36(4), 717-731. https://doi.org/10.3758/BF03206553

Preacher, K. J., \& Hayes, A. F. (2008). Asymptotic and resampling strategies for assessing and comparing indirect effects in multiple mediator models. Behavior research methods, 40(3), 879-891. https://doi.org/10.3758/BRM.40.3.879 